\title{
Spectral and luminosity classification for the cool components in symbiotic stars
}

\author{
Z.X. Zhu ${ }^{1,4}$, M. Friedjung ${ }^{2}$, G. Zhao ${ }^{3,4}$, H.R. Hang ${ }^{1,4}$, and C.C. Huang ${ }^{1,4}$ \\ 1 Purple Mountain Observatory, 210008 Nanjing, PR China \\ e-mail: zxzhu@mail.pmo.ac.cn \\ 2 Institute d'Astrophysique, 98 bis Boulevard, 75014 Paris, France \\ e-mail: fried@iap.fr \\ 3 Beijing Astronomical Observatory, Datun Road 20A, 100012, PR China \\ 4 National Astronomical Observatories, Chinese Academy of Sciences, PR China
}

Received May 20; accepted September 15, 1999

\begin{abstract}
The near infrared spectra of 12 S-type symbiotic stars and 78 comparison stars have been observed with moderate dispersion in five runs from 1992 to 1997, the resolving power being $R=\frac{\lambda}{\Delta \lambda}>2000$, with a signal to noise ratio $S / N>100$. The triple-headed absorption band of $\mathrm{TiO}(\lambda \lambda 8432,8422$ and $8452 \AA$ ) emerges when a star is later than M2, and the depth of the TiO absorption band is very sensitive to the spectral type (ST) and insensitive to the luminosity class of the star. We fit a curve of spectral type against the index of the absorption depth of this band with a standard deviation $\sigma=0.37$ of a subdivision of one spectral type. The IR CaII triplet $(\lambda \lambda 8498,8542,8662 \AA)$, Fe I $8689 \AA$, and Fe I $8675 \AA$ are good luminosity indicators although the equivalent widths (EWs) of these lines clearly decrease for a star later than M3. When the star is a supergiant, the lines have a smaller central residual intensity and broader wings than in the case of a normal giant. The Ca II $8662 \AA / F e$ I $8675 \AA$ and Fe I $8689 \AA /$ Fe I $8675 \AA$ ratios are also good luminosity indicators for K-type giants. The latter is particularly useful when there are abundance anomalies. The metal-poor symbiotic star AG Dra is classified as a Ib or II giant, as is TX CVn, on the basis of Fe I $8689 \AA$ A/Fe I $8675 \AA$. 9 other symbiotic stars containing M-type cool components are classified as giants by direct comparison and quantitative analysis. Due to there being no known good ratio indicator of luminosity for M-type stars in the band studied and because there is no metal abundance data for the symbiotic stars studied by us except for AG Dra, the results for these 9 symbiotic stars are only preliminary. The infrared Ca II triplet of most symbiotic stars clearly varies between the different observing runs. The different luminosity classes given to the same symbiotic star are probably caused by the variability of the lines of ionized
\end{abstract}

elements, while in some cases they are affected by a low metal abundance.

Key words: stars: binaries: symbiotic — stars: fundamental parameters - stars: individual: TX CVn, T CrB, AG Dra - infrared: stars

\section{Introduction}

Symbiotic stars are interacting binaries consisting of three components: one is the compact stellar component, which may be either a white dwarf or a main sequence star or a neutron star; the other cool stellar component is a giant or a supergiant, which can be sometimes a Mira variable. The third component is a kind of nebula surrounding both of these stellar components. Knowing the properties of the cool component gives important constraints on the nature of the binary system; in particular the light curve of the cool component can at least sometimes be related to the change of the rate of transfer of material to the compact component needed to "activate" the latter. If we can accurately determine the spectral type and luminosity class of the cool component, we shall be able to obtain better information on its evolutionary status and also derive properties of the hot companion (Kenyon \& Webbink 1984), so enabling us to deduce more realistic models for symbiotic stars including those needed to describe the processes which occur during their activity stages.

The spectral type mainly represents the surface temperature of the star. The luminosity class represents the absolute luminosity and the geometry scale of the star. 
It also includes information about the evolutionary status of the star. So, the luminosity classification of the cool components in symbiotic stars plays an important role in the field of symbiotic stars. However, luminosity classification should be based on accurate spectral type classification.

There are two spectral classification systems, the Harvard system and the Yerkes system (known as MKK and MK). Only small differences exist between them. Spectral classification is performed mainly from the strengths of lines, including especially the ratios of line intensities. Traditionally classification was based on the wavelength range $(\lambda \lambda 3500-4900 \AA)$ to which early plates were sensitive. Now higher dispersions and broader wavelength ranges are used due to the development of technology. Classification ought to be carried out under the same instrumental conditions.

For the late, $\mathrm{K}$ and $\mathrm{M}$ type stars, there are many features which can be used to make a classification of spectral type and luminosity class, these being described in the book "The Classification of Stars", by Jaschek \& Jaschek (1987). Keenan \& Hynek (1945), Sharpless (1956), Ramsey (1981) and Bell \& Tripicco (1991) have given many spectral and luminosity criteria in the optical and infrared bands. If the spectral type is earlier than M5, the following luminosity indicators can be used: Sr II $\lambda 4077 / \mathrm{Fe}$ I $\lambda 4263$, (Y II + Fe I) $\lambda 4376 / \mathrm{Fe}$ I $\lambda 4383, \mathrm{Fe}$ II $\lambda 7712 / \mathrm{Ni}$ II $\lambda 7714$, Fe I $\lambda 8689 / \mathrm{Fe}$ I $\lambda 8675, \mathrm{CN} \lambda \lambda 7878$, 8044, 8068, etc. Solf (1978), Diaz et al. (1989) and Zhou (1991) also pointed that the IR CaII triplet and some FeI and TiI lines are good luminosity indicators except for very late (>M3) stars; however, the spectral types of most of the symbiotic stars are later than M3 (Medina Tanco \& Steiner 1995; Muerset \& Schmid 1999).

Due to the orbital motion of the binary, the spectral classification of the cool components in symbiotic stars is complicated by the variable superimposed blue continuum and the reflection effect. The great differences between the spectral types and luminosity classes determined by different authors for the same symbiotic star have confused the "picture" made of the star; for example, AG Dra has been classified as G5III (Mirozoyan et al. 1960), G7III (Smith Bopp 1981), K0Ib (Huang 1982; Huang et al. 1994), K1 (Allen 1982), K3III (Boyarchuck 1966), earlier than K4 III (Kenyon \& Fernandez-Castro 1987) and K5III (Belyakina 1969); such differences could be partly caused by the variability of the star.

Early spectral classifications of symbiotic cool components were collected by Allen (1982). Kenyon \& Gallagher (1983) used low resolution observations to obtain a [2.35]-[2.2] colour index, depending on the intensity of the $2.35 \mu \mathrm{m}$ CO band, which is a luminosity class discriminator. This was followed up by a new lower resolution infrared photometric study of Kenyon (1988), which was found unsuitable for determining photometrically the $\mathrm{CO}$ strength.
Kenyon \& Fernande-Castro (1987) performed important work on the temperature classification of symbiotic stars by using the depths of $\mathrm{TiO}$ and $\mathrm{VO}$ bands, but there are no very good luminosity sensitive features in the band $(\lambda \lambda 7600-8400 \AA)$ they observed for the full range of temperature; the luminosity classes they found by visually comparing various features, are not very certain. SchulteLadbeck (1988) performed the temperature classification of cool components in the Sharpless (1956) system by visually looking at tracings of Reticon spectra; the luminosity class could be found for only 6 objects up to a spectral type of M4 from the sum of the equivalent widths of the CN 7916, $7941 \AA$ bands.

In later papers Huang et al. (1994), Medina Tanco \& Steiner (1995), Harries \& Howarth (1997), Mikolajewska Acker and Stenholm and finally Muerset \& Schmid (1999) have, in particular, also made contributions to spectral classification for the cool component in symbiotic stars. Medina Tanco and Steiner had problems using the indices of Kenyon (1986) and Kenyon \& Fernandez-Castro (1987), so they combined them with visual comparisons of standard spectra, the last being used for fine tuning of the temperature classifications they obtained. Mikolajewska et al. (1997) determined the same indices as Kenyon and Fernandez-Castro, which they found not completely reliable. Muerset \& Schmid (1999) found temperature classes only.

The literature does not deny the presence of more evolved components than giants in some S-type symbiotic stars. In general they appear to have larger mass loss rates compared with those of single giants. Medina Tanco \& Steiner (1995) selected the symbiotic stars in the direction of the galactic bulge in order to draw the $\mathrm{H}-\mathrm{R}$ diagram for the stars, assuming that the stars have similar distances from the earth. Their results demonstrate that the cool components of the symbiotic stars are more evolved than normal giants (in the thermally pulsing AGB region). Muerset et al. (1996) obtained a similar result for symbiotic stars of the Magellanic Clouds and in particular for S type cool components of the LMC. One may wonder whether such results involving the excessive luminosities of certain populations of cool components of symbiotics, are partly caused by selection effects.

In order to confirm and improve the previous spectral classification and look for a solution which can lead to a more satisfactory luminosity classification, we observed the infrared spectra of 12 symbiotic stars and 78 comparison stars in five runs in spectral regions between $7900 \AA$ and $9400 \AA$. Spectral classification should be more reliable in this wavelength band than at shorter wavelengths, because the influences of the hot companion and the surrounding nebula are smaller there. Let us note also that no detailed analysis has been performed up to now of the atmosphere of any cool symbiotic component of spectral type M, which is the spectral type in most cases. 


\section{Observations and data reduction}

CCD spectra were obtained with the $1.93 \mathrm{~m}$ telescope of the Haute Provence Observatory and the $2.16 \mathrm{~m}$ telescope in the XingLong station of the Beijing Observatory. A journal of observations is listed in Table 1; the dispersion and the resolution obtained from the FWHM of the lines of the calibration lamp are also given in addition. The data were reduced by the IRAF software mounted on a SUN station. The standard spectral reduction procedure was used. The main parameters involved are indicated later when needed. No correction for atmospheric extinction and no flux calibration were made.

When the wavelength is less than $8400 \AA$, the atmospheric absorption lines are strong and must be eliminated. In order to do this each spectrum was divided by an atmospheric spectrum. The sky absorption spectrum was taken as equal to $F=\left(F_{\mathrm{s}}-1\right) \frac{A M}{A M_{\mathrm{s}}}+1$. Where $F_{\mathrm{s}}$ is the normalized sky absorption spectrum obtained from the standard early type stars. $A M$ and $A M_{\mathrm{s}}$ are the air mass of the star and standard star respectively. Because of variations of water vapor density in the earth's atmosphere (i.e. $\frac{A M}{A M_{\mathrm{s}}}$ is variable), we take a $\frac{A M}{A M_{\mathrm{s}}}$ value near 1 and make several attempts for each spectrum observed until the resulting spectrum is satisfactory.

\section{Spectral type classification}

Merrill et al. (1962) have pointed out that the absorption depth of $\mathrm{TiO}$ and $\mathrm{VO}$ varies with $T_{\mathrm{e}}$. We define the index of the depth of the triple-headed absorption band of $\mathrm{TiO}$ as O'Connell (1973) has done: $[\mathrm{TiO}]=-2.5 \log \left(F_{\lambda} / F_{\mathrm{c}}\right)$, where $F_{\lambda}$ is the flux at $\lambda$ and $F_{\mathrm{c}}$ is the interpolated continuum flux at $\lambda$. The continuum was produced by the "continuum" IRAF program. The main parameters are as follows: the left windows - $8390-8410 \AA$; the right window - $8700-8725 \AA$; lower points with more than a $1 \sigma$ deviation below the continuum are eliminated, while the order of the fitting function is 1 (see Fig. 1), the dashed line being the resulting continuum. The spectrum was divided by this continuum to be normalized, then we can measure $F_{\lambda} / F_{\mathrm{c}}$ directly; the window for measuring $F_{\lambda} / F_{\mathrm{c}}$ is from $8455 \AA$ to $8470 \AA$, points more than $2 \sigma$ below the mean level in this window are eliminated (i.e. the effect of the metal absorption lines is eliminated). The windows for making the continuum and measuring $\mathrm{F} \lambda / F_{\mathrm{c}}$ are marked in Fig. 1. the $[\mathrm{TiO}]$ indices of the comparison stars are given in the last column of Table 2 and Table 3 ; when it is empty the $\mathrm{TiO}$ absorption band does not emerge in the star and this also means the star is earlier than M3.

Figure 2 shows the spectral type of the comparison stars against the $[\mathrm{TiO}]$ index. From Fig. 2 we see that $[\mathrm{TiO}]$ is sensitive to the spectral type and insensitive to the luminosity class. The relationship between the spec-

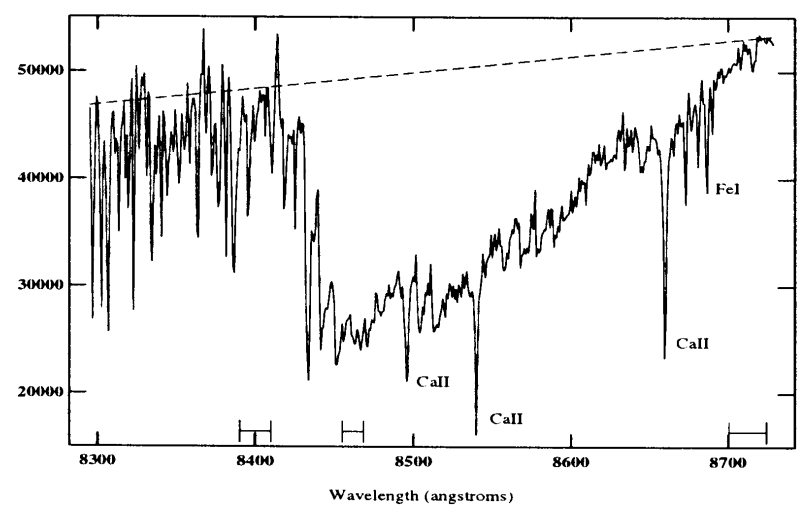

Fig. 1. How to measure the $F_{\lambda} / F_{\mathrm{c}}$. The windows for making the continuum and the window for measuring $F_{\lambda} / F_{\mathrm{c}}$ are marked. The dashed line is the artificial continuum

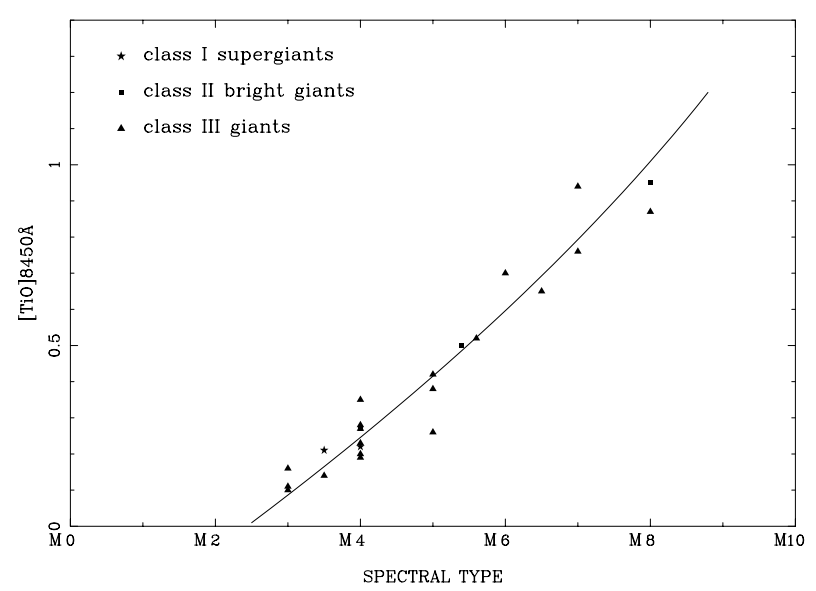

Fig. 2. ST vs. the $[\mathrm{TiO}]$ index. The curve is the fitted line. For $\mathrm{M} 0, \mathrm{ST}=0 ;$ for $\mathrm{M} 6, \mathrm{ST}=6$

tral type $\mathrm{ST}$ and $[\mathrm{TiO}]$ has been obtained by least squares fitting to be:

$\mathrm{ST}=2.43+6.65[\mathrm{TiO}]-1.12[\mathrm{TiO}]^{2}$

where at $\mathrm{M} 0$, the value of $\mathrm{ST}=0$, while for $\mathrm{M} 10, \mathrm{ST}=10$. The standard deviation is only 0.37 , the curve in Fig. 2 is drawn from the fitted function, it shows the depth of the triple-headed absorption band of $\mathrm{TiO}$ is a very good temperature indicator.

This TiO band emerges in 8 of the 12 symbiotic stars, the $[\mathrm{TiO}]$ indices being given in Table 4 . Columns 2 to 5 correspond to the different observing runs. The [TiO] index varies with the time of observation, due to the reflection effect, which is correlated with the orbital motion of the binary. The variation of the spectral type calculated by Eq. (1) is 0.8 of a subdivision for CH Cyg and 0.5 for BF Cyg and CI Cyg, which are similar to or less than for the results calculated from other molecular bands by Kenyon \& Fernandez-Castro (1987). This may be due to the observations being made at only two or three epochs for each star and the longer wavelength of the band. The latest types are given in the last column of Table 4. 
Table 1. The observing journal and technical parameters

\begin{tabular}{cccccc}
\hline Observing run & Date & Telescope & Band & Dispersion & Resolution \\
\hline 1 & $1992.6 .12-6.15$ & $1.93 \mathrm{~m}$ & $8100-8630 \AA$ & $33 \AA / \mathrm{mm}$ & $2.0 \AA$ \\
2 & $1993.5 .30-6.1$ & $1.93 \mathrm{~m}$ & $8100-8740 \AA$ & $33 \AA / \mathrm{mm}$ & $2.0 \AA$ \\
3 & $1996.5 .29-5.30$ & $2.16 \mathrm{~m}$ & $8380-9400 \AA$ & $50 \AA / \mathrm{mm}$ & $3.5 \AA$ \\
4 & 1997.7 .13 & $2.16 \mathrm{~m}$ & $7900-9000 \AA$ & $50 \AA / \mathrm{mm}$ & $3.5 \AA$ \\
5 & $1997.11 .7-11.8$ & $2.16 \mathrm{~m}$ & $8000-9000 \AA$ & $50 \AA / \mathrm{mm}$ & $3.5 \AA$ \\
\hline
\end{tabular}

Table 2. The data for the comparison stars observed at $33 \AA / \mathrm{mm}$ in 1993

\begin{tabular}{|c|c|c|c|c|c|c|c|c|c|c|c|c|c|c|}
\hline \multirow[t]{2}{*}{ Name } & \multirow{2}{*}{$\begin{array}{l}\text { Sp. type } \\
\AA)\end{array}$} & \multicolumn{2}{|c|}{$\mathrm{EW}(\mathrm{NaI})$} & \multicolumn{3}{|c|}{$\mathrm{EW}(\mathrm{CaII})$} & \multicolumn{2}{|c|}{$\mathrm{EW}(\mathrm{FeI})$} & \multicolumn{5}{|c|}{ EW(TiI) } & \multirow{2}{*}{$\begin{array}{l}\mathrm{TiO} \\
8450\end{array}$} \\
\hline & & 8183 & 8194 & 8498 & 8542 & 8662 & 8675 & 8689 & 8364 & 8378 & 8382 & 8397 & 8412 & \\
\hline HR 5200 & K5III & & & 1.99 & 4.14 & 3.13 & 0.45 & 0.49 & 0.47 & 0.25 & 0.63 & 0.25 & 0.44 & \\
\hline HR 5631 & KOIII & 0.24 & 0.26 & 1.68 & 4.07 & 2.70 & 0.24 & 0.39 & 0.28 & 0.11 & 0.26 & 0.07 & 0.19 & \\
\hline HR 5854 & K2IIIb & & & 1.95 & 4.42 & 3.11 & 0.38 & 0.69 & 0.35 & 0.38 & 0.24 & 0.66 & 0.38 & \\
\hline HR 5879 & M0.5IIIab & & & 1.96 & 3.88 & 3.25 & 0.48 & 0.61 & 0.53 & 0.35 & 0.71 & 0.29 & 0.41 & \\
\hline HR 6119 & M7III & & & & & & & & & & & & & 0.94 \\
\hline HR 6495 & M4IIIab & 0.40 & 0.56 & 1.44 & 3.12 & 3.19 & 0.55 & 0.67 & 0.56 & 0.40 & 0.62 & 0.37 & 0.46 & 0.35 \\
\hline HR 6498 & K2II & 0.23 & 0.31 & 2.02 & 5.07 & 3.67 & 0.51 & 0.71 & 0.29 & 0.31 & 0.57 & 0.24 & 0.37 & \\
\hline HR 6702 & M5.6III & 0.37 & 0.53 & 1.04 & 2.75 & 2.75 & 0.41 & 0.58 & 0.52 & 0.37 & 0.59 & 0.34 & 0.40 & 0.52 \\
\hline HR 6705 & K5III & & & 2.27 & 5.04 & 3.70 & 0.54 & 0.73 & 0.58 & 0.34 & 0.68 & 0.23 & 0.38 & \\
\hline HR 7157 & M5III & 0.36 & 0.56 & 1.07 & 2.91 & 3.18 & 0.53 & 0.65 & 0.56 & 0.38 & 0.59 & 0.38 & 0.40 & 0.50 \\
\hline HR 7475 & $\mathrm{~K} 4 \mathrm{Ib}$ & 0.25 & 0.27 & 2.38 & 6.26 & 4.58 & 0.86 & 0.96 & 0.75 & 0.54 & 0.78 & 0.39 & 0.54 & \\
\hline HR 7506 & G8III & 0.24 & 0.24 & 1.81 & 3.85 & 2.81 & 0.32 & 0.47 & 0.18 & 0.14 & 0.20 & 0.12 & 0.11 & \\
\hline HR 7509 & M5IIIa & & & 1.23 & 3.00 & 3.13 & 0.52 & 0.65 & 0.56 & 0.39 & 0.61 & 0.38 & 0.45 & 0.42 \\
\hline HR 7523 & M3III & 0.33 & 0.43 & 1.63 & 3.30 & 2.72 & 0.45 & 0.49 & 0.35 & 0.26 & 0.48 & 0.22 & 0.31 & 0.16 \\
\hline HR 7525 & K3II & 0.24 & 0.20 & 2.29 & 5.20 & 3.91 & 0.62 & 0.75 & 0.55 & 0.42 & 0.64 & 0.24 & 0.31 & \\
\hline HR 7568 & M4IIb & 0.55 & 0.72 & 1.50 & 3.55 & 3.44 & 0.62 & 0.78 & 0.58 & 0.40 & 0.62 & 0.38 & 0.51 & 0.27 \\
\hline HR 7831 & K2III & 0.13 & 0.29 & 1.80 & 3.93 & 3.02 & 0.40 & 0.61 & 0.31 & 0.25 & 0.34 & 0.16 & 0.23 & \\
\hline HR 7866 & $\mathrm{~K} 2 \mathrm{Ib}$ & 0.24 & 0.27 & 2.33 & 5.91 & 4.60 & 0.85 & 0.96 & 0.74 & 0.47 & 0.73 & 0.35 & 0.46 & \\
\hline HR 7886 & M6III & 0.34 & 0.41 & 0.95 & 1.78 & 1.67 & 0.33 & 0.41 & 0.41 & 0.45 & 0.47 & 0.41 & 0.31 & 0.70 \\
\hline HR 7921 & G8IIb & 0.16 & 0.28 & 2.05 & 4.32 & 3.43 & 0.36 & 0.47 & 0.24 & 0.14 & 0.24 & 0.14 & 0.19 & \\
\hline HR 7951 & M3III & & & 1.63 & 3.83 & 3.24 & 0.48 & 0.62 & 0.53 & 0.37 & 0.58 & 0.35 & 0.42 & 0.10 \\
\hline HR 8079 & K4Ib-II & 0.54 & 0.65 & 2.10 & 5.41 & 4.09 & 0.75 & 0.60 & 0.37 & 0.67 & 0.32 & 0.45 & & \\
\hline HR 8225 & M1III & 0.33 & 0.56 & 2.14 & 5.44 & 4.24 & 0.67 & 0.83 & 0.50 & 0.34 & 0.56 & 0.24 & 0.29 & \\
\hline HR 8465 & $\mathrm{~K} 1.5 \mathrm{Ib}$ & & & 2.63 & 5.55 & 4.52 & 0.69 & 0.92 & 0.51 & 0.37 & 0.68 & 0.32 & 0.51 & \\
\hline HR 8726 & $\mathrm{~K} 5 \mathrm{Ib}$ & 0.65 & 0.78 & 2.60 & 5.53 & 4.35 & 0.69 & 0.86 & 0.65 & 0.39 & 0.73 & 0.38 & 0.62 & \\
\hline RW Cyg & M3.5I & 0.41 & 0.73 & 1.40 & 3.81 & 3.67 & 0.64 & 0.84 & 0.67 & 0.47 & 0.65 & 0.46 & 0.62 & 0.21 \\
\hline BI Cyg & M4I & & & 1.74 & 3.85 & 3.93 & 0.78 & 1.01 & 0.85 & 0.56 & 0.75 & 0.48 & 0.77 & 0.22 \\
\hline
\end{tabular}

References for Sp. type:

The Bright Star Catalogue (Hoffleit 1982).

Johnson $(1965,1968)$.

Taylor (1986).

These values should be the most insensitive to the reflection effect.

UV Aur has been considered as a C star, which is suspicious because of the emergence of TiO. A C star normally has no TiO band due to very poor Oxygen.

\section{Luminosity classification}

According to Diaz et al. (1989) and Zhou (1991) the infrared Ca II triplet, and a doublet of $\mathrm{Na}$ I can be used for luminosity classification. Unfortunately the lines of the Ca II triplet tend to be blended with Paschen emission lines in the spectra of symbiotic stars and they are also diluted by the emission due to the influence of the hot component, while the strengths of the lines studied are also sensitive to metal abundance. We therefore seek to use other lines.

The measured EWs of all the strong lines of the comparison stars are listed in Table 2 and Table 3 . Table 2 lists the data obtained from the spectra taken in France 
Table 3. The data of the comparison stars observed with $50 \AA / \mathrm{mm}$

\begin{tabular}{|c|c|c|c|c|c|c|}
\hline \multirow[t]{2}{*}{ Name } & \multirow{2}{*}{$\begin{array}{l}\text { Sp. type } \\
(\AA)\end{array}$} & \multicolumn{3}{|c|}{ EW(CaII) } & \multirow{2}{*}{$\begin{array}{l}\mathrm{EW}(\mathrm{FeI}) \\
8689\end{array}$} & \multirow{2}{*}{$\begin{array}{l}{[\mathrm{TiO}]} \\
8450\end{array}$} \\
\hline & & 8498 & 8542 & 8662 & & \\
\hline HR 112 & K0IV & 1.46 & 3.37 & 2.43 & 0.23 & \\
\hline HR 134 & KOIII & 1.95 & 3.81 & 2.92 & 0.35 & \\
\hline HR 175 & G8III & 1.77 & 3.51 & 2.99 & 0.29 & \\
\hline HR 344 & MOIII & 2.04 & 4.40 & 3.82 & 0.44 & \\
\hline HR 355 & M1III & 1.92 & 4.36 & 3.61 & 0.70 & \\
\hline HR 511 & K0V & 1.39 & 3.09 & 2.31 & 0.20 & \\
\hline HR 568 & K0IV & 1.61 & 3.08 & 2.59 & 0.34 & \\
\hline HR 601 & M2III & 1.95 & 3.84 & 3.24 & 0.65 & \\
\hline HR 738 & KOIII & 1.59 & 3.69 & 2.87 & 0.45 & \\
\hline HR 750 & M3IIIa & 2.07 & 4.12 & 3.17 & 0.56 & \\
\hline HR 753 & K3V & 1.25 & 3.14 & 2.30 & 0.32 & \\
\hline HR 969 & G6Ib-IIa & 1.96 & 4.44 & 3.55 & 0.28 & \\
\hline HR 978 & G8IV & 1.22 & 2.64 & 2.00 & 0.50 & \\
\hline HR 995 & G8IV & 1.23 & 3.03 & 2.63 & 0.28 & \\
\hline HR 1048 & KOIV & 1.35 & 3.17 & 2.49 & 0.27 & \\
\hline HR 1601 & $\mathrm{~K} 2 \mathrm{II}$ & 2.08 & 4.45 & 3.94 & 0.25 & \\
\hline HR 1255 & KOIII-IV & 1.48 & 3.08 & 2.21 & 0.32 & \\
\hline HD 224126 & M7-10III & & & & & 0.87 \\
\hline HD 10465 & M2Ib & 2.57 & 5.19 & 4.60 & 0.88 & \\
\hline HD 1013 & M2III & 2.07 & 4.14 & 3.46 & 0.56 & \\
\hline HR 743 & G8III & 1.72 & 3.39 & 2.81 & 0.25 & \\
\hline HD 27022 & G5IIb & 1.76 & 3.69 & 2.91 & 0.14 & \\
\hline HD 29139 & K5III & 2.00 & 4.36 & 3.16 & 0.57 & \\
\hline HD 37160 & K0IIIb & 1.20 & 3.00 & 2.47 & 0.51 & \\
\hline HR 2063 & M6.5IIIe & & & & & 0.65 \\
\hline HR 2048 & G9II & 2.33 & 4.60 & 4.22 & 0.39 & \\
\hline HR 1925 & $\mathrm{~K} 1 \mathrm{~V}$ & 1.39 & 3.27 & 2.26 & 0.54 & \\
\hline HR 1688 & K2III-IV & 1.70 & 3.61 & 2.81 & 0.48 & \\
\hline HR 2153 & G9III & 1.57 & 3.38 & 2.66 & 0.35 & \\
\hline HR 2747 & M4IIIab & 1.83 & 3.71 & 2.91 & 0.65 & 0.23 \\
\hline HR 3061 & M4III & 1.64 & 2.99 & 2.85 & 0.38 & 0.28 \\
\hline HD 44478 & M3IIIab & 2.01 & 3.75 & 3.43 & 0.56 & 0.11 \\
\hline HD 52497 & G5Ib-IIa & 2.11 & 4.54 & 3.77 & 0.34 & \\
\hline HR 4956 & K4III & 1.85 & 4.21 & 2.70 & 0.46 & \\
\hline HR 5226 & M3.5III & 1.71 & 3.79 & 2.89 & 0.77 & \\
\hline HR 4964 & K3III & 1.79 & 3.74 & 2.34 & 0.59 & \\
\hline HR 5053 & K0III & 1.41 & 3.28 & 2.45 & 0.47 & \\
\hline HR 5145 & K3III & 1.78 & 3.78 & 2.51 & 0.55 & \\
\hline HR 5200 & K5III & 1.97 & 4.13 & 2.71 & 0.66 & \\
\hline HR 5300 & M1.5III & 1.78 & 3.91 & 2.86 & 0.69 & \\
\hline HR 5340 & K1IIIb & 1.57 & 3.57 & 2.33 & 0.68 & \\
\hline HR 5352 & M3IIIa & 1.88 & 3.95 & 3.01 & 0.82 & \\
\hline HR 5490 & M3III & 1.88 & 3.92 & 2.72 & 0.69 & \\
\hline HR 5589 & M5III & 1.32 & 3.01 & 2.59 & 0.55 & 0.26 \\
\hline HR 5631 & K0III & 1.66 & 3.82 & 2.61 & 0.31 & \\
\hline HR 5654 & M4IIIab & 1.55 & 3.58 & 2.87 & 0.64 & 0.19 \\
\hline HR 5854 & K2IIIb & 1.78 & 3.74 & 2.46 & 0.56 & \\
\hline HR 5879 & M0.5IIIab & 1.68 & 3.93 & 2.86 & 0.56 & \\
\hline HR 5924 & MOIII & 1.75 & 3.90 & 2.76 & 0.69 & \\
\hline HR 6495 & M4IIIab & 1.53 & 3.21 & 2.69 & 0.82 & 0.27 \\
\hline HR 6498 & K2II & 2.12 & 4.64 & 3.59 & 0.62 & \\
\hline HR 6705 & K5III & 2.12 & 4.56 & 3.79 & 0.77 & \\
\hline HR 7183 & M3.5IIIab & 1.52 & 3.55 & 2.80 & 0.88 & 0.14 \\
\hline HR 7157 & M5III & 1.19 & 2.60 & 2.40 & 0.69 & 0.38 \\
\hline HR 7328 & G9III & 1.80 & 3.60 & 2.73 & 0.37 & \\
\hline HR 7412 & $\mathrm{~K} 5 \mathrm{Ib}$ & 2.41 & 4.92 & 3.74 & 0.88 & \\
\hline HR 7475 & $\mathrm{~K} 4 \mathrm{Ib}$ & 2.48 & 5.36 & 4.01 & 0.95 & \\
\hline HR 9079 & G9III-IV & 1.73 & 3.43 & 3.07 & 0.18 & \\
\hline HR 9099 & M4III & 1.87 & 3.47 & 2.88 & 0.62 & 0.20 \\
\hline Y Uma & M7II-III & & & & & 0.76 \\
\hline RX Boo & M8e & & & & & 0.95 \\
\hline
\end{tabular}

with a $33 \AA / \mathrm{mm}$ dispersion. Table 3 lists the corresponding results from observations with a $50 \AA / \mathrm{mm}$ dispersion. Due to the blending of the weak lines, only the
Table 4. The [TiO] index in the different runs and ST of symbiotic objects

\begin{tabular}{llllll}
\hline Symbiotic & \multicolumn{4}{c}{$[\mathrm{TiO}]$} & ST \\
\hline obs. run & 2 & 3 & 4 & 5 & \\
\hline YY Her & 0.00 & 0.12 & & & M3.2 \\
CH Cyg & 0.67 & 0.73 & 0.56 & & M6.7 \\
CI Cyg & 0.31 & & 0.25 & & M4.4 \\
AG Peg & 0.00 & & 0.00 & & $<$ M3 \\
Z And & 0.18 & & & & M3.6 \\
T CrB & 0.21 & 0.16 & & & M3.8 \\
V443 Her & 0.40 & 0.37 & & & M4.9 \\
BF Cyg & 0.52 & & 0.42 & & M5.6 \\
UV Aur & & & & 0.35 & C ?? \\
\hline \hline
\end{tabular}

strongest lines are measured on $50 \AA / \mathrm{mm}$ spectra, these being the Ca II triplet and Fe I $8689 \AA$. There is an approximately $10 \%$ systematic deviation between the EWs of the two sets of data, having different dispersions. The continua for measuring the EWs were deduced with the help of the "continuum" program in IRAF, the parameters used in "continuum" being: spline3 function, the order is 1 , points with deviations of more than $1 \sigma$ below the continuum are eliminated, while those with $3 \sigma$ above the continuum are eliminated in the spectra of symbiotic stars in order to remove the emission lines. Due to the fact that the different radial velocities of the stars produce different shifts of the lines and that many metal lines fill this band, taking fixed windows to measure EWs will result in large deviations. We must therefore measure the lines one by one carefully. We estimate the deviation in $\mathrm{EW}$ to be $10 \%$ for the CaII triplet, $15 \%$ for Fe I $8689 \AA$, $20 \%$ for Ti I and more than $25 \%$ for $\mathrm{Na}$ I. The graph of the total EW of Ca II $8542 \AA$ and Ca II $8662 \AA$ is plotted against spectral type ST in Fig. 3a, where $\mathrm{ST}$ is defined so that for G9, ST $=-7$; for $\mathrm{K} 0$, ST $=-6$, while for $\mathrm{K} 6$ and $\mathrm{M} 0$, ST $=0$. All the $50 \AA / \mathrm{mm}$ data in Table 3 are used regardless of whether the same stars are listed in different parts of the table. There are different equivalent widths for stars which have the same spectral type and a different luminosity class. Figure 4 shows that the Ca II of supergiants has the least central residual intensity and the broadest wings among the K2 type standard stars, this being also true for the Fe I $8689 \AA$ and Fe I $8675 \AA$ lines. When ST > 3 (later than M3), the EWs clearly decrease, but still there is difference between those stars having the same spectral type. The central residual intensity of Ca II is also seen to be different (see Fig. 5). Comparing the spectra of T Cr B and of the type I, II and III giants, we can easily conclude that the luminosity class of the cool component of $\mathrm{T} \mathrm{CrB}$ is III.

Figure $3 \mathrm{~b}$ is the graph of the EW of Fe I $8689 \AA$ against ST, Fig. 3c is for the total EW of all measured Ti I lines, while Fig. 3d is that for the $\mathrm{Na} 8183 \AA$ plus Na I $8194 \AA$ lines. The EWs of the Fe I, Ti I and Na I lines 

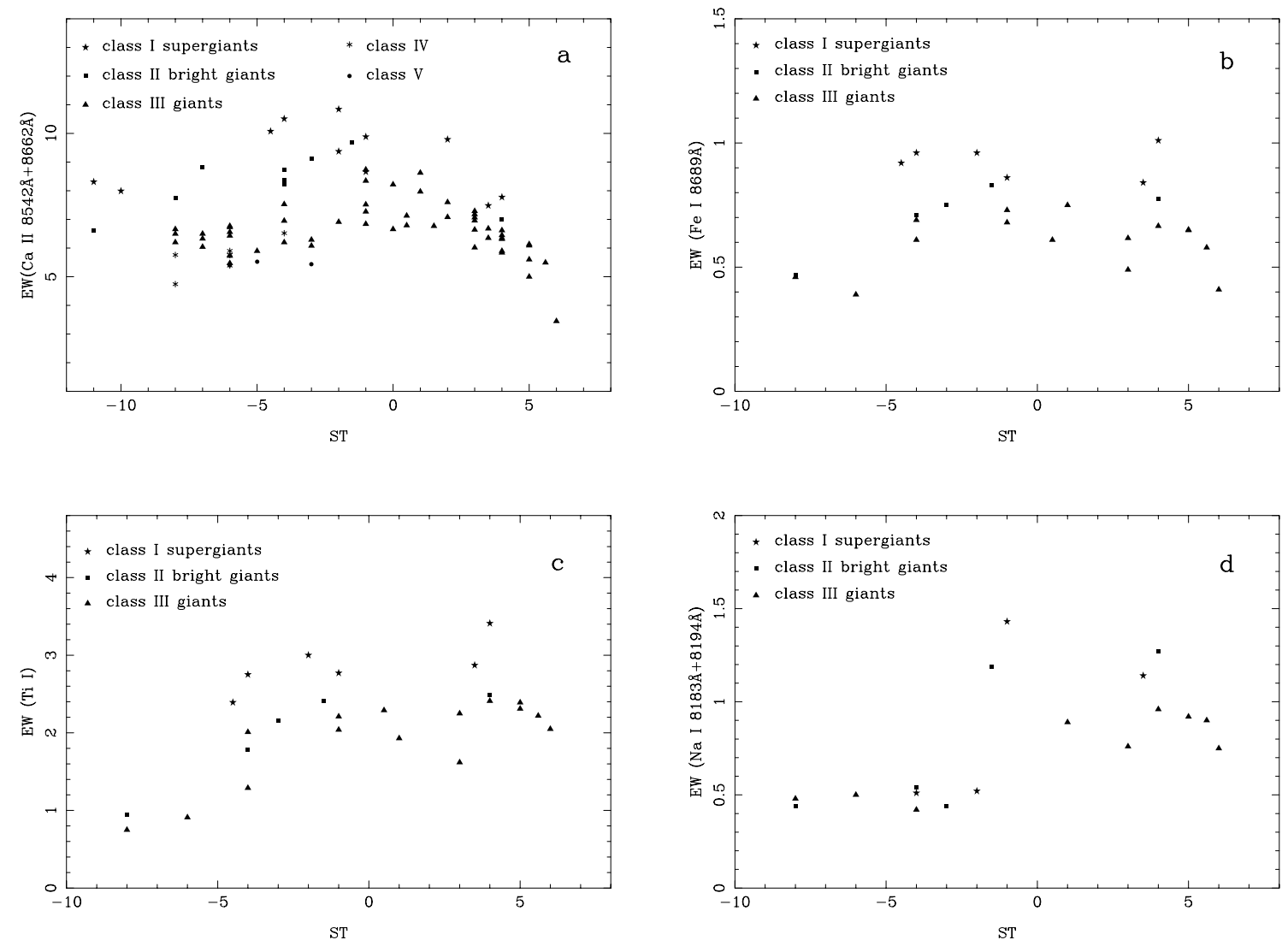

Fig. 3. ST vs. EW. For G9, ST $=-7$; for K0, ST $=-6$; for $\mathrm{K} 6$ and M0, ST =0. a) vs. Ca II $8542 \AA+8662 \AA$; b) vs. Fe I $8689 \AA$; c) vs. all measured Ti I; d) vs. Na I $8183 \AA+8194 \AA$

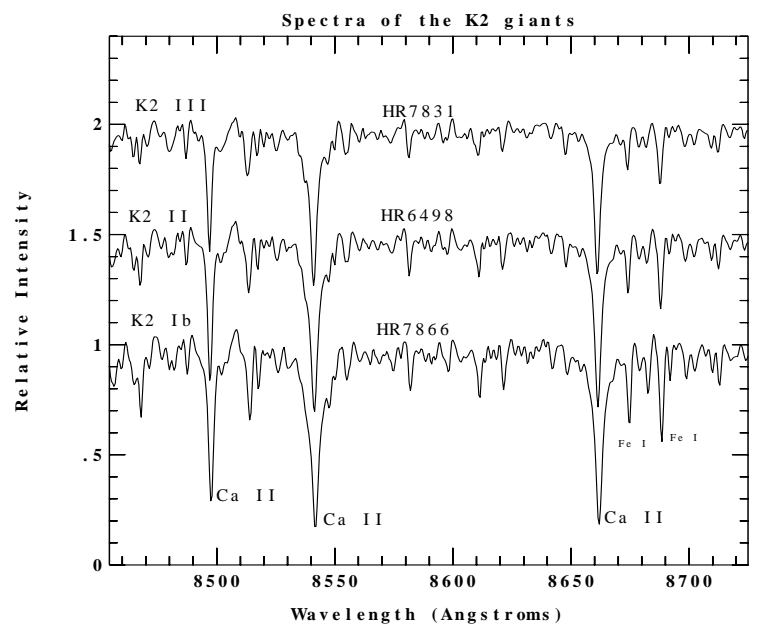

Fig. 4. The spectra of K2-type giants

are those measured only in the comparison spectra with a $33 \AA / \mathrm{mm}$ dispersion, so there are fewer points in the last three graphs. In particular Fe I $8689 \AA$ is affected by blending in the $50 \AA / \mathrm{mm}$ spectra. We find that Fe I $8689 \AA$ is a good luminosity indicator, so is Ti I, but not $\mathrm{Na}$ I. Perhaps the measurement deviation of $\mathrm{Na}$ I is too large.
In fact Jaschek \& Jaschek (1987) pointed out that Fe I $8689 / F e$ I 8675 is a luminosity indicator for M-type stars. Due to the lack of metal abundance determinations for most symbiotic stars, it is much better to use the ratio of these lines to determine the luminosity class. Their closeness in wavelength minimizes moreover effects due to varying contributions at different wavelengths of residual continuum emission of the hot compoment. This ratio vs. $\mathrm{ST}$ is shown in Fig. 6; however we only find that it is a luminosity indicator for K-type stars. The ratio Ca II 8662 to Fe I 8675 against ST is shown in Fig. 7, it being also an indicator for K-type stars. Nevertheless we must be cautious to use it because of the chemical anomalies of some stars such as those of many symbiotic stars (Smith et al. 1996, 1997; Pereira 1995). Another reason is that CaII $8662 \AA$ of a symbiotic star may be diluted by the emission due to the presence of the hot star.

The EWs of the symbiotic stars with $33 \AA / \mathrm{mm}$ spectra taken in 1993 are listed in Table 5; the EW value followed by a $\mathrm{B}$ character means that the line is blended with some visible emission, while a negative EW value means that the line is in emission. The data for the $50 \AA / \mathrm{mm}$ dispersion spectra are listed in Table 6. We observed most symbiotic stars twice and the EWs of the atomic lines do not vary greatly. 

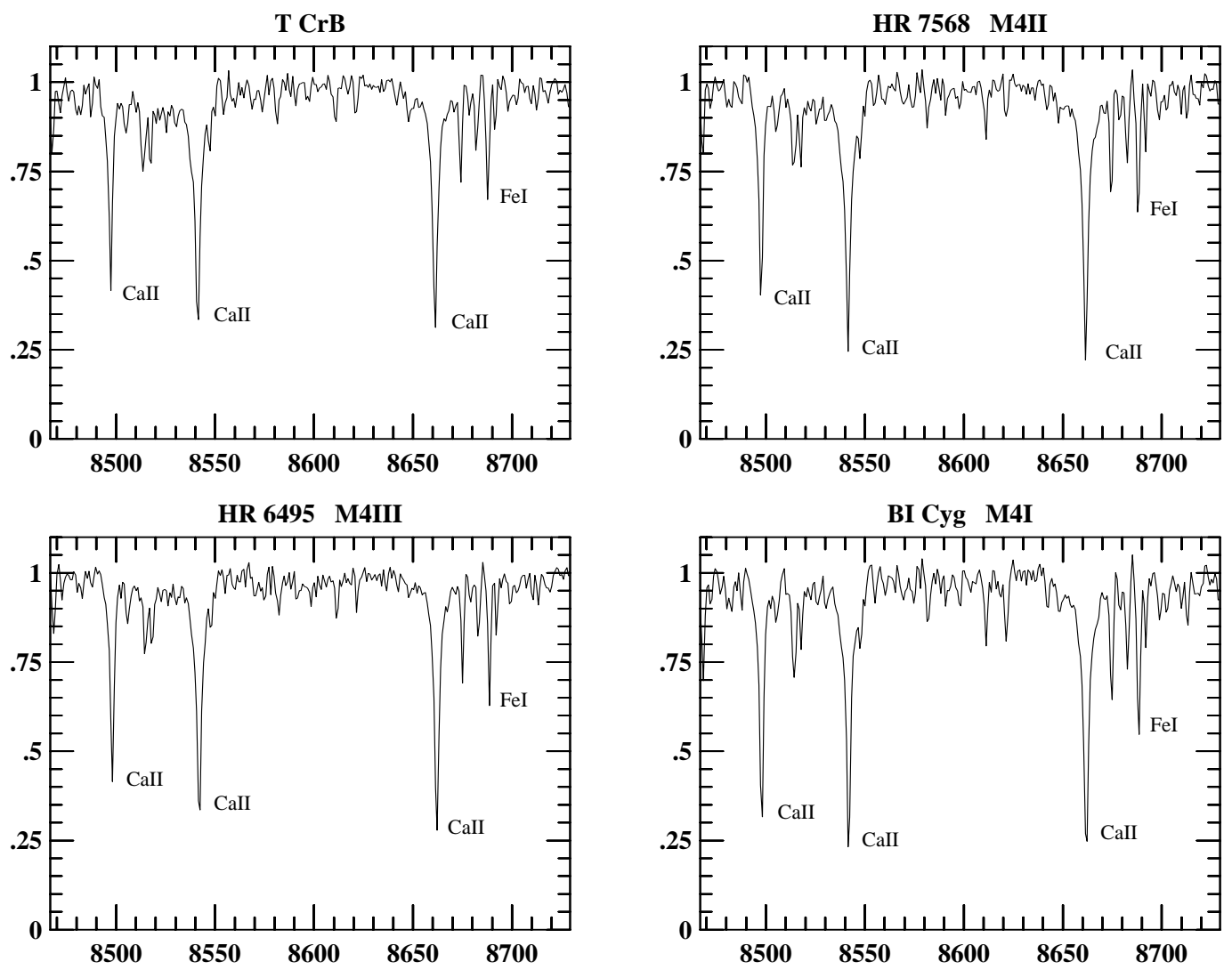

Fig. 5. The spectra of $\mathrm{T} \mathrm{CrB}$ and the comparison giants

However the Ca II triplets of some symbiotic stars vary dramatically, presumably due to emission produced by the presence of the hot companion. Therefore, Fe I $8689 \AA$ is the best luminosity indicator for symbiotic objects as long as one can assume solar abundances for metals, while the ratio indicator Fe I $8689 / \mathrm{Fe}$ I 8675 is a better luminosity indicator, usable for K-type symbiotic cool components.

Figure 8 is the normalized spectrum of AG Dra. The Ca II triplet lines are blended with the Paschen emission lines of hydrogen and possibly some Ca II emission due to the presence of the hot component. If we assume that P13, P14, P15, P16, P17 have the same EW, then the EW of Ca II with the Paschen lines minus the average EW of P14 and P17 is the real EW of Ca II. The EWs of $\mathrm{Ca}$ II and Fe I are much less than those of type III giants. The very detailed stellar atmosphere analysis of Smith et al. (1996) shows that AG Dra is a metal-poor giant, $\left([\mathrm{Fe} / \mathrm{H}]=-1.3, \log (g)=1.6, T_{\text {eff }}=4300 \mathrm{~K}\right)$. Therefore, it is more suitable to use the ratio luminosity indicator to determine its luminosity class. Fe I 8689/Fe I 8675 and CaII 8662/Fe I 8675 are 1.12 and 6.38 respectively for AG Dra. From Fig. 6 AG Dra is classified as a supergiant (the spectral type of AG Dra is supposed to be K0-K3). The effect of the metal abundance must be considered, when using the latter indicator, if the star is very metal-poor. The low metal abundance results in a lower electron pres- sure and more ions (Saha formula). For the very metalpoor star AG Dra, Ca II 8662/Fe I 8675 should then be larger than for stars with a normal metal abundance, if only simple Saha equation effects are taken in account. In addition we must also take into account Smith's result, that $[\mathrm{Ca} / \mathrm{Fe}]=0.21$. The ratio Ca II $8662 \AA / \mathrm{Fe}$ I 8675 $\AA$ of this star would be less than 6.38 , if that abundance ratio were normal. Comparing with Fig. 7, we obtain in fact the result that the lines have a ratio characteristic of a Ib or a II giant. The result obtained by the latter indicator is not certain, as Ca II $8662 \AA$ may be diluted by the emission due to the presence of the hot stellar component. However AG Dra may still also have a larger luminosity and a larger radius than a normal giant, and indeed Smith et al. (1996) admit that their determination of the physical parameters of the cool component is not completely precise. Physically it would be easier to understand the processes of AG Dra if it were larger than a normal giant (Huang et al. 1994). The minimum distance of $1 \mathrm{kpc}$ from HIPPARCOS observations found by Viotti et al. (1997) is unfortunately not decisive, as the maximum $M_{v}$ near 1.0 is compatible with the cool component being a class III giant.

The corresponding ratios for TX CVn are 1.02 and 6.10 respectively, giving a luminosity class for the cool component of this system of II or Ib from Fig. 6 and Fig. 7, taking into consideration that the spectral type for TX 


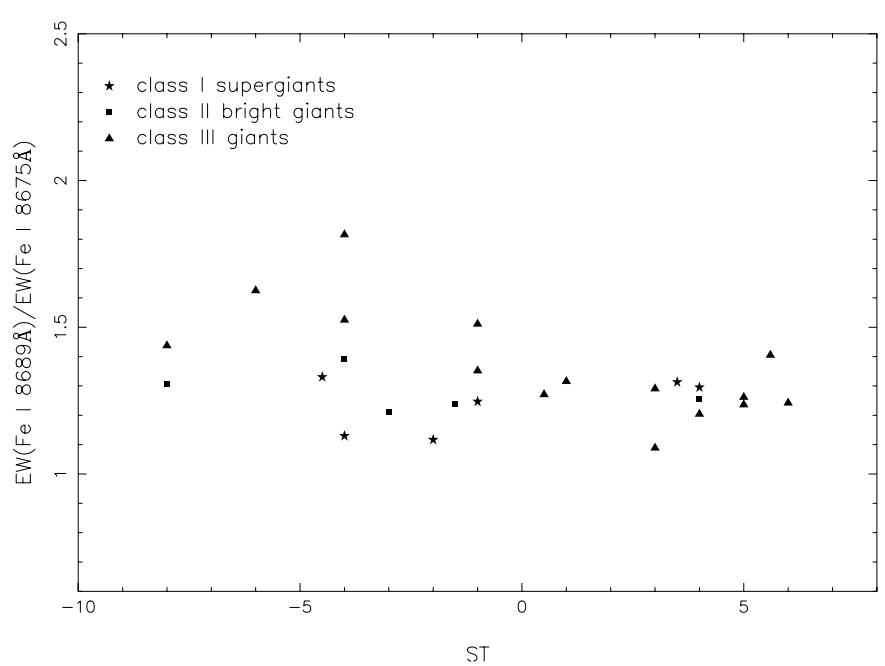

Fig. 6. ST vs. Fe I $8689 \AA / F e$ I $8675 \AA$

CVn is K5.3 according to Kenyon \& Fernandez-Castro (1987). The same uncertainty exists for this object as for AG Dra concerning conclusions obtained from Fig. 7, because of emission due to the presence of the hot component. Kenyon \& Garcia (1989) determined orbital solutions for this binary. They suggest that the components are a K5 III + a B9 shell star, the orbital period being 199 days. However they admit that there are quite many uncertainties. According to them the hot component is not a normal B9 star, but could rather be a white dwarf, undergoing a hydrogen shell flash. The cool component could, using their conditions for the orbit, be in addition up to almost 50 times brighter than a K5 III giant, without necessarily exceeding the size of its orbit. The exact limit for the cool component not to be larger than its Roche lobe clearly depends on the masses assumed for each stellar component.

Finally, it must also be pointed out that the ratio indicator of luminosity is not very sure because of the small number of standard stars. The present ratio indicators are moreover suitable only for K-type stars.

The luminosity classes of the other symbiotic stars are determined from the EW of Fe I $8689 \AA$, Ti I and Ca II. F I $8689 \AA$ plays the key role. We compare the central residual intensities and the wings of Fe I $8689 \AA$ and the Ca II triplet of the symbiotic stars with the standard stars. The spectral types of most symbiotic stars are taken from Table 4; those of AG Peg and EG And being taken as M3.0 and M2.4 (Kenyon \& Fernandez-Castro 1987). The lines of the symbiotic stars are usually similar to those of a giant or a little weaker, except that Ca II of some symbiotic stars is in emission. The results of luminosity classification for the symbiotic stars are given in the last column of Table 6 . The visual comparison gives the final result, and Fe I $8689 \AA$ has an important role, except for AG Dra and TX CVn where the ratio criteria are used.

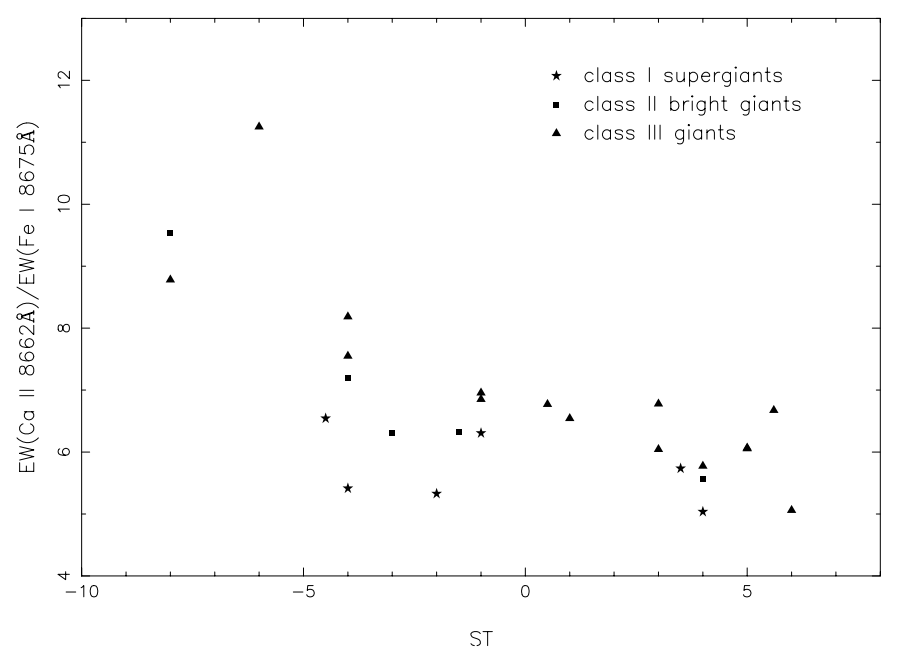

Fig. 7. ST vs. Ca II $8662 \AA / F e$ I $8675 \AA$

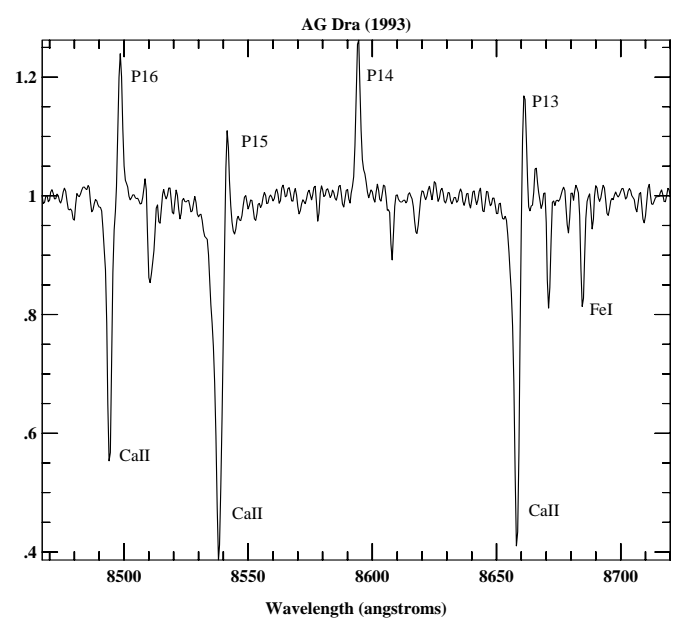

Fig. 8. The normalized spectrum of AG Dra

\section{Discussion and conclusions}

The triple-headed absorption band of TiO near $8450 \AA$ is a good temperature indicator for the late type stars (later than M2). Our spectral type results for the symbiotic stars are consistent with those of Kenyon \& Fernandez-Castro (1987), and the spectral types of the different symbiotic stars vary within the limits of $0.3-0.8$ subdivision due to binary motion. After obtaining an accurate spectral type of each symbiotic star, visual comparison and comparisons using quantities connected with the Fe I $8689 \AA$ and Ca II triplet with standard stars which have the same spectral type, lead to the luminosity classes of the symbiotic stars being obtained.

The graph EW vs. color index $(R-I)$ has been drawn, but there is no obvious difference in EWs between different type giants when the spectral type is later than M3; perhaps the $\mathrm{ST}$ is a better temperature indicator than $(R-I)$. 
Table 5. The data of 1993

\begin{tabular}{cccccccccc}
\hline Symbiotic star & \multicolumn{3}{c}{ EW(CaII) } & \multicolumn{1}{c}{ EW $(\mathrm{FeI})$} & \multicolumn{5}{c}{ EW(TiI) } \\
\hline$\lambda \lambda(\AA)$ & 8542 & 8662 & 8675 & 8689 & 8364 & 8378 & 8382 & 8379 & 8412 \\
\hline AG Dra & $3.11 \mathrm{~B}$ & $2.17 \mathrm{~B}$ & 0.34 & 0.38 & 0.18 & 0.18 & 0.37 & 0.13 & 0.11 \\
YY Her & $-0.74 \mathrm{~B}$ & $-0.43 \mathrm{~B}$ & 0.47 & 0.52 & 0.41 & 0.24 & 0.39 & 0.19 & 0.27 \\
CH Cyg & 0.76 & 1.32 & 0.30 & 0.41 & 0.37 & 0.32 & 0.44 & 0.36 & 0.27 \\
TX CVn & 3.26 & 2.50 & 0.41 & 0.42 & 0.35 & 0.24 & 0.45 & 0.19 & 0.34 \\
CI Cyg & 1.82 & 2.33 & 0.49 & 0.59 & 0.55 & 0.44 & 0.58 & 0.31 & 0.37 \\
AG Peg & 3.79 & 2.65 & 0.31 & 0.43 & 0.48 & 0.31 & 0.60 & 0.30 & 0.29 \\
Z And & $1.28 \mathrm{~B}$ & $1.22 \mathrm{~B}$ & 0.43 & 0.52 & 0.53 & 0.37 & 0.60 & 0.31 & 0.33 \\
T CrB & 3.14 & 3.19 & 0.49 & 0.69 & 0.53 & 0.38 & 0.64 & 0.35 & 0.43 \\
V443 Her & $0.64 \mathrm{~B}$ & $1.30 \mathrm{~B}$ & 0.42 & 0.50 & 0.53 & 0.41 & 0.59 & 0.42 & 0.28 \\
BF Cyg & $1.38 \mathrm{~B}$ & $1.55 \mathrm{~B}$ & 0.42 & 0.51 & 0.61 & 0.42 & 0.59 & 0.41 & 0.45 \\
\hline
\end{tabular}

Table 6. The data of the $50 \AA / \mathrm{mm}$ spectra

\begin{tabular}{|c|c|c|c|c|c|c|c|}
\hline Date & & $996,5,29-3$ & & & $1997,7,13$ & & \\
\hline Symbiotic star & EW(CaII) & EW(CaII) & $\mathrm{EW}(\mathrm{FeI})$ & $E W(C a I I)$ & $E W(C a I I)$ & EW(FeI) & $\mathrm{LC}$ \\
\hline$\lambda \lambda(\AA)$ & 8542 & 8662 & 8689 & 8542 & 8662 & 8689 & \\
\hline AG Dra & $2.46 \mathrm{~B}$ & $1.85 \mathrm{~B}$ & 0.38 & & & & Ib or II \\
\hline YY Her & 2.28 & 1.72 & 0.35 & & & & IIIb \\
\hline $\mathrm{CH} \mathrm{Cyg}$ & 1.26 & 1.34 & 0.40 & 1.15 & 1.37 & 0.32 & III \\
\hline TX CVn & 3.06 & 2.16 & 0.40 & & & & $\mathrm{II}$ or $\mathrm{Ib}$ \\
\hline CI Cyg & & & & 1.54 & 1.37 & 0.58 & III or IIIa \\
\hline AG Peg & & & & 2.75 & 1.98 & 0.59 & III \\
\hline $\mathrm{Z}$ And & & & & $-1.86 \mathrm{~B}$ & $-1.86 \mathrm{~B}$ & 0.66 & III \\
\hline $\mathrm{T} \mathrm{CrB}$ & 2.79 & 2.15 & 0.45 & & & & III \\
\hline V443 Her & 0.91 & 1.25 & 0.32 & & & & III \\
\hline BF Cyg & & & & $1.56 \mathrm{~B}$ & 1.69B & 0.56 & III \\
\hline EG And* & 3.76 & 2.82 & 0.40 & & & & III \\
\hline
\end{tabular}

* EG And was only observed on 7/11/97.

The Ca II infrared triplet is a good luminosity indicator, but for most symbiotic stars Ca II varies dramatically because of the emission due to the hot component of symbiotic binaries. Therefore we must be very careful about using these lines for symbiotic binaries.

Fe I $8689 \AA$ is a good luminosity indicator for latetype stars including symbiotic stars. Ca II 8662/Fe I 8675 and Fe I $8689 / \mathrm{Fe}$ I 8675 are luminosity indicators for K-type stars. Fe I 8689/Fe I 8675 may be the best for symbiotic stars due to the chemical composition anomalies and the emission produced by the hot component. This indicator shows that AG Dra and TX CVn have an apparently brighter luminosity class than that of giants.

If classification is made by comparing Fe I $8689 \AA$ and the Ca II triplet, AG Dra and TX CVn could be classified as normal giants, but their ratios to Fe I $8675 \AA$ are those of more luminous stars. One major reason at least is that AG Dra is very metal-poor. The question arises whether we can suppose TX CVn to be also metal-poor. However its low systemic radial velocity of $2.3-2.5 \mathrm{~km}$ $\mathrm{s}^{-1}$ (Kenyon \& Garcia 1989), is not one characteristic of metal poor stars. We might suspect nevertheless that all symbiotic stars have abnormal metal abundances and if that is the case, there are questions also concerning the luminosity class results for those symbiotic stars whose cool companions are M-type giants.

Acknowledgements. The authors gratefully acknowledge the generous allocations of the observation time of the telescopes in Haute-Province Observatory and Xinglong Station; gratefully acknowledge the help of Dr. J.Y. Wei and the observing assistants during observation; gratefully acknowledge the helpful suggestions of the referee J.E. Steiner. This research has made use of NASA's Astrophysics Data System Abstract Service.

\section{References}

Allen D.A., 1982, in "The Nature of Symbiotic Stars", Friedjung M. and Viotti. R. (eds.), p. 27

Ander E., Grevess N., 1989, Geochim. Cosmochim. Acta 53, 197 
Bell R.A., Tripicco M.J., 1991, AJ 102, 777

Belyakina T.S., 1969, Izv. Krym. Astrophys. Obs. 40, 39

Boyarchuk A.A., 1966, Astrofizica 2, 101

Diaz A.I., Terlevich E., Terlevich R., 1989, MNRAS 239, 325

Harries T.J., Howarth I.D., 1996, A\&AS 119, 61

Hoffleit D., 1982, "The Bright Star Catalogue", Fourth Revised Edition. Yale University Observatory

Huang C.C., 1982, in "The Nature of Symbiotic Stars", Friedjung M. and Viotti R. (eds.). Reidel, p. 185

Huang C.C., Friedjung M., Zhu Z.X., 1994, A\&AS 106, 413

Jaschek C., Jaschek M., 1987, in "The Classification of Stars". Cambridge University Press, p. 354

Johnson H.L., 1965, ApJ 141, 170

Johnson H.L., 1968, ApJ 152, 465

Keenan P.C., Hynek J.A., 1945, ApJ 101, 265

Kenyon S.J., 1986, "The Symbiotic Stars". Cambridge University Press, p. 45

Kenyon S.J., 1988, AJ 96, 337

Kenyon S.J., Fermandez-Castro T., 1987, AJ 93, 938

Kenyon S.J., Gallagher J.S., 1983, AJ 88, 666

Kenyon S.J., Garcia M.R., 1989, AJ 97, 194

Kenyon S.J., Webbink R.F., 1984, ApJ 279, 252
Medina Tanco G.A., Steiner J.E., 1995, AJ 109, 1770

Merrill P.W., Deutsch A.J., Keenan P.C., 1962, ApJ 136, 21

Mikolajewska J., Acker A., Stenholm B., 1997, A\&A 327, 191

Mirozoyan L.V., Bartaya R.A., 1960, Izv. Ambast. Astrophys. Obs. 25, 121

Muerset U., Schild H.M., 1999, A\&AS 137, 473

Muerset U., Schild H.M., Vogel M., 1996, A\&A 307, 516

O'Connell R.W., 1973, AJ 78, 1074

Pereira C.B., 1995, A\&AS 111, 471

Ramsey L.W., 1981, AJ 86, 557

Sharpless S., 1956, ApJ 124, 342

Schulte-Ladbeck R.E., 1988, A\&A 189, 97

Smith S.E., Bopp B.W., 1981, MNRAS 195, 733

Smith V.V., Cunha K., Jorissen A., Boffin H.M.J., 1996, A\&A 315, 179

Smith V.V., Cunha K., Jorissen A., Boffin H.M.J., 1997, A\&A 324,97

Solf J., 1978, A\&AS 34, 409

Taylor B.J., 1986, ApJS 60, 577

Viotti R., Badiali M., Cardini D., Emanuele A., Iijima T., 1997, ESA SP-402, 405

Zhou X., 1991, A\&A 248, 367 